\title{
Rebrote arbóreo en la regeneración del bosque tropical de Calakmul, Campeche, México
}

\author{
María Alejandra Haas-Ek ${ }^{1}$, Noel Antonio González-Valdivia2*,
} Bernardus Hendricus Jozeph De Jong ${ }^{1}$, Susana Ochoa-Gaona ${ }^{1}$, \& Deb Raj Aryal ${ }^{3}$

1. Departamento de Ciencias de la Sustentabilidad, El Colegio de la Frontera Sur, México; mahaas28568@gmail.com, bjong@ecosur.mx, sochoa@ecosur.mx

2. Tecnológico Nacional de México, Instituto Tecnológico de Chiná, Departamento de Ingenierías; siankaan2003@gmail.com

3. CONACYT-Facultad de Ciencias Agronómicas, Universidad Autónoma de Chiapas, México; debraj.aryal@hotmail.com

* Correspondencia

Recibido 07-V-2018. Corregido 16-IX-2018. Aceptado 21-I-2019.

\begin{abstract}
Arboreal resprouting in the Calakmul, Campeche, Mexico tropical forest regeneration. Detailed studies to understand recovering of Neotropical forests over time, through secondary succession, are still necessaries. Between acting mechanisms of regeneration in the tropical forests, the arboreal resprout predominates in woody species, with the advantage of restart the growth from still rooting plants, allowing the restoring of forest canopy. The study aim was to identify and quantify the tree species with resprouting capacity in different stages of secondary succession (tree-fallow) after the traditional milpa agricultural system, compared with tropical forest communities in the Calakmul Biosphere Reserve and, enlightening how these species influences the recovery of the composition of this ecosystem. Was calculated the proportions of species with or without resprouting by successional stage. There were 13972 individuals (32\% resprouting and $68 \%$ not resprouting) and 168 species (79\% resprouting and $21 \%$ not resprouting), the percentage of individuals regrowing decreases as the successional stage progresses. The number of individuals resprouting and species composition were significantly different between successional stages. In conclusion, mostly arboreal species in the region of Calakmul, can regrow, which influences the successional development of the forest after slash-and-burn, helping to restore the coverage, structure, as well as species composition original.
\end{abstract}

Key words: secondary succession; arboreal species; resprouting; species composition; tropical forest.

Haas-Ek, M. A., González-Valdivia, N. A., De Jong, B. H. J., Ochoa-Gaona, S., \& Aryal, D. R. (2019). Rebrote arbóreo en la regeneración del bosque tropical de Calakmul, Campeche, México. Revista de Biología Tropical, 67(1), 164-181.

Los estudios detallados para comprender cómo se reestablecen los bosques neotropicales a través de la sucesión secundaria son necesarios para sistematizar suficientes conocimientos para manejarlos y conservarlos de manera más integral. Aunque los bosques secundarios ocupan del 30-50 \% del total de las masas forestales en el mundo, existen relativamente pocos estudios sobre su dinámica sucesional (Brown \& Lugo, 1990; Guariguata \& Ostertag, 2001; Chazdon et al., 2009; Barbosa, Broadbent, \& Bitencourt, 2014). La comprensión de los mecanismos que actúan en la sucesión de estos bosques es fundamental para el desarrollo de estrategias para su conservación y su restauración (Baider, Tabarelli, \& Mantovani, 
2001; Quesada et al., 2009). Algunos aspectos estudiados e importantes para estos fines incluyen predominantemente a los estudios sobre sucesión, composición y estructura florística (Rico-Gray, García-Franco, Puch, \& Simá, 1988; Zamora-Crescencio et al., 2011; Dupuy et al., 2012; Dzib-Castillo, ChanatásigVaca, \& González-Valdivia, 2014), y menos frecuentemente a otros elementos como los mecanismos de dispersión (González-Valdivia et al., 2012), ambiente y dinámica de recursos (Fahey \& Puettmann, 2007; Aryal, de Jong, Ochoa-Gaona, Esparza-Olguín, \& MendozaVega, 2014). Procesos de sucesión relacionados con el rebrote son poco conocidos.

Varios autores señalan que entre los mecanismos de regeneración de los bosques tropicales el rebrote de árboles es característico de las especies leñosas (Baider et al., 2001; Benítez-Malvido, Martínez-Ramos, \& Ceccon 2001; Poorter et al., 2010). Esto posibilita el reinicio del bosque a partir del crecimiento desde raíces establecidas, lo que permite restituir rápidamente la composición original del dosel forestal (Gómez-Pompa \& VázquezYanes, 1985; Ochoa-Gaona \& GonzálezEspinosa, 2000; Paciorek, Condit, Hubbell, \& Foster, 2000; ITTO, 2002; Ochoa-Gaona, Hernández-Vásquez, de Jong, \& Gurri-García, 2007). El proceso de sucesión depende de la disponibilidad de tocones, propágulos, el tipo de vegetación, regímenes de disturbio e intensidad del uso del suelo por intervención humana (González-Iturbe, Olmsted, \& Tun-Dzul, 2002; Barbosa et al., 2014).

En paisajes fragmentados o sujetos a perturbaciones periódicas, el rebrote, como atributo de resiliencia, posibilita la regeneración forestal como una posible respuesta adaptativa que facilita la recuperación del bosque (Yhi, Boucher, Vandermeer, \& Zamora, 1991; Granda-Moser, Finegan, Ramos-Bendaña, Detlefsen, \& Molina, 2015; Poorter et al., 2016). Este fenómeno ha sido relativamente poco estudiado en los bosques y en la sucesión forestal, con algunos esfuerzos investigativos de la sucesión post-agrícola (Uhl, 1987; Paciorek et al., 2000; Boucher et al., 2001; Ferguson \& Griffith, 2004).

En la Península de Yucatán convergen disturbios naturales, como los huracanes, sequías e incendios, y antropogénicos, como las actividades agrícolas. Dentro de este último, destaca el sistema agrícola tradicional, conocido como milpa, caracterizado por la corta de árboles y su posterior quema, dejando tocones que se dejan rebrotar, lo que facilita la recuperación de la cobertura durante el barbecho (Islebe et al., 2009; Carrillo-Bastos, Elizalde-Rendón, Torrescano-Valle, \& Flores-Ortiz, 2008). La coexistencia de ambos procesos de disturbio ha permitido la selección de especies adaptadas que facilitan la regeneración rápida de estos bosques (Levy-Tacher \& Hernández-Xolocotzi, 1992). Los huracanes rompen los tallos y derriban los árboles, pero estos reinician su crecimiento a partir de los fustes rotos o tumbados. La biomasa acumulada por derribos de ramas y árboles, es combustible que en condiciones de sequía, fenómeno frecuente en esta región, propicia el fuego forestal. El humano, con la implementación del sistema de agroforestal rotacional que tradicionalmente se conoce como milpa, corta los árboles, luego los quema y establece cultivos anuales por dos o tres ciclos anuales. Estos tres procesos han actuado para, posiblemente, seleccionar especies que, después de recibir daños mecánicos a sus fustes y/o quemarse, reinician su crecimiento desde el tocón o remanente de tallo o incluso raíz.

La generación de información sobre el rebrote de especies arbóreas permitirá mejorar la comprensión de cómo este mecanismo contribuye a la regeneración de los bosques tropicales. Este es el motivo principal para la realización de este estudio, que tuvo como objetivo identificar y cuantificar el papel de las especies arbóreas con capacidad de rebrote en distintas etapas de la sucesión secundaria después del sistema agrícola tradicional de milpa y compararlas con las especies de comunidades del bosque tropical predominante en la Reserva de la Biósfera de Calakmul, y cómo influyen estas especies en la recuperación de la composición de estos bosques. 


\section{MATERIALES Y MÉTODOS}

Área de estudio: El área de estudio se ubica en la Reserva de la Biósfera de Calakmul (REBICA), al sureste del Estado de Campeche (Fig. 1), abarcando cuatro ejidos: Nuevo Conhuás (NC, $18^{\circ} 27^{\prime} 50.4$ " $\mathrm{N} \& 89^{\circ} 53$ ' 23.5 " W), Cristóbal Colón $\left(\mathrm{CC}, 18^{\circ} 10^{\prime} 46.3\right.$ " $\mathrm{N} \& 89^{\circ}$ $27^{\prime} 36.3^{\prime \prime} \mathrm{W}$ ), El Carmen II (EC, $18^{\circ} 09^{\prime} 48.2^{\prime \prime}$ $\mathrm{N} \& 89^{\circ} 23$ ' 56.3" W ) y Narciso Mendoza (NM, $\left.18^{\circ} 15^{\prime} 40.1 " \mathrm{~N} \& 89^{\circ} 27^{\prime} 25.2 " \mathrm{~W}\right)$. El clima de la REBICA es cálido subhúmedo con lluvias en verano, el promedio de precipitación anual es de $1076.2 \mathrm{~mm}$ y la temperatura promedio anual es de $24.6{ }^{\circ} \mathrm{C}$. Los suelos que se desarrollan son leptosoles, gleysoles y vertisoles. Con respecto a la hidrología, la región presenta cuerpos de agua naturales (aguadas) y lagunetas artificiales de recolección de agua de lluvia, denominadas localmente "jagüeyes" (INE, 2000).

El tipo de vegetación dominante es el boque tropical subhúmedo, conocido como selva mediana subperennifolia (Pennington \& Sarukhán, 2005). En esta selva, entre las especies más importantes se encuentran: Manilkara zapota (zapote), Brosimum alicastrum (ramón),
Bucida buceras (pucté), Lonchocarpus castilloi (machiche) con especies asociadas como: Pouteria reticulata (zapotillo), Bursera simaruba (chaca), Ceiba pentandra (ceiba) y Vitex gaumeri (yaax nic), entre otros (Martínez-Salas \& Galindo-Leal, 2002).

Diseño de muestreo: Se utilizaron 39 parcelas permanentes $(20 \times 50 \mathrm{~m})$ que forman parte de un estudio a largo plazo sobre dinámica forestal y de carbono, establecido por El Colegio de la Frontera Sur en el municipio de Calakmul, estado de Campeche, México, que además proveyó la base información sobre la historia de uso que cada parcela recibió (Aryal et al., 2014; García-Licona, Esparza-Olguín, \& Martínez-Romero, 2014). Las parcelas se asignaron de manera que representaran cuatro etapas sucesionales que van desde el bosque secundario joven hasta el bosque tropical maduro (Cuadro 1). Todas las parcelas de vegetación secundaria provienen del sistema agrícola tradicional de roza-tumba y quema (milpa). Algunas parcelas permanentes previamente establecidas, no pudieron ser incluidas en este estudio, debido a que habían sido destruidas

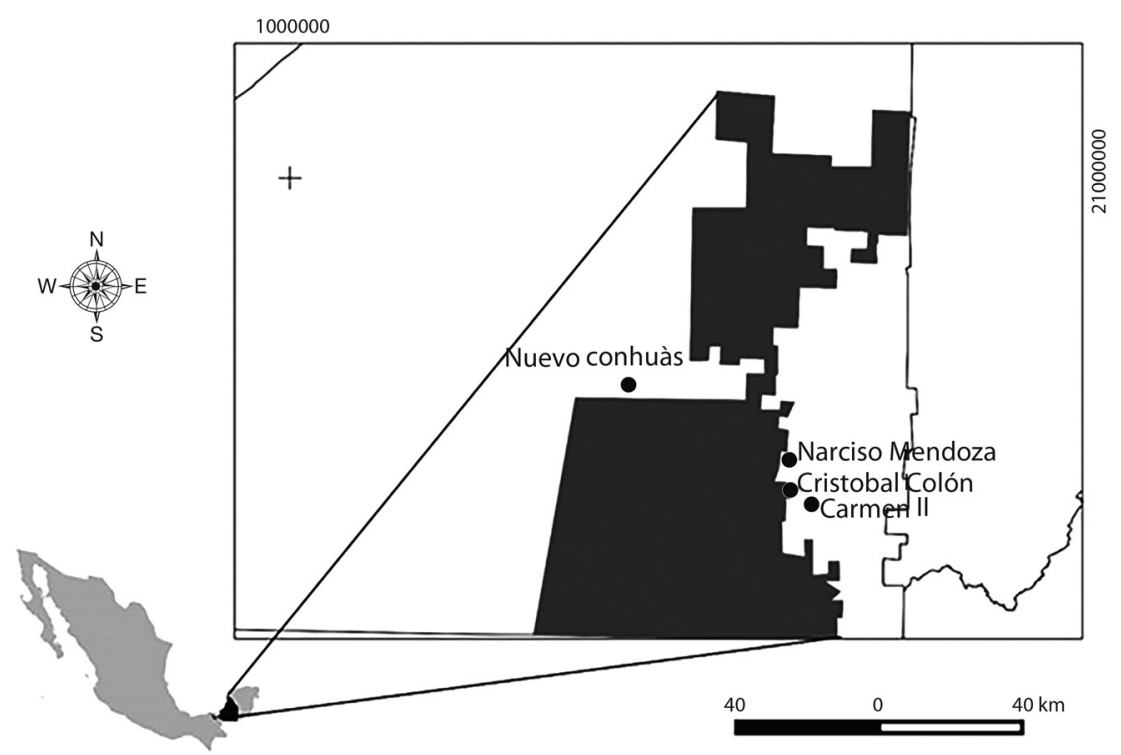

Fig. 1. Sitios estudiados en el área de la Reserva de la Biósfera de Calakmul, Campeche, México.

Fig. 1. Location of the sites studied in the area of the Biosphere Reserve of Calakmul, Campeche, Mexico. 
CUADRO 1

Clasificación de las etapas sucesionales para el bosque tropical, con el número de parcelas que representó cada una en el bosque tropical de Calakmul, Campeche, México

TABLE 1

Classification of the successional stages for the tropical forest, with the number of plots that each represented in the tropical forest of Calakmul, Campeche, Mexico

\begin{tabular}{lcc}
\multicolumn{1}{c}{ Etapa sucesional } & Edad sucesional & No. de parcelas \\
Bosque Secundario Joven (BSJ) & 4 y 10 años & 10 \\
Bosque Secundario Intermedio (BSI) & 16 y 20 años & 11 \\
Bosque Secundario Avanzado (BSA) & 26 y 40 años & 12 \\
Bosque Tropical (BT) & más de 115 años & 6 \\
\hline
\end{tabular}

por cambios de uso de suelo, por tanto no fue posible uniformar el tamaño de muestra para cada etapa sucesional. No obstante, cada clase tuvo entre 9 y 12 repeticiones, similar a lo establecido en estudios de la sucesión en Calakmul por Román-Dañobeytia, Levy-Tacher, Macario-Mendoza y Zúñiga-Morales (2014). La distancia mínima entre parcelas fue de $1000 \mathrm{~m}$.

\section{Composición florística y estructura:} Cada parcela de muestreo incluyó una parcela grande de $20 \times 50 \mathrm{~m}\left(1000 \mathrm{~m}^{2}\right)$, con una parcela interior de $10 \times 40 \mathrm{~m}\left(400 \mathrm{~m}^{2}\right)$. En la parcela de grande se muestrearon todos los individuos leñosos con DAP (diámetro a la altura del pecho a $1.30 \mathrm{~m}$ sobre el suelo) $\geq 5$ $\mathrm{cm}$ y, en la parcela interior todos los individuos leñosos con DAP $\geq 1 \mathrm{~cm}$. De cada árbol fue medido el DAP con cinta diamétrica, la altura con ayuda de una escala telescópica graduada y se contó el número de individuos por cada parcela. La identificación de las especies arbóreas se realizó con ayuda de claves dicotómicas y un guía local (parataxónomo). Para la actualización de los nombres científicos fueron consultadas las páginas The Plant List (2013) y Tropicos.org (2018).

Rebrote: Para este estudio se consideró como rebrote a la presencia, desde una base común, generalmente un tocón, de más de un tallo en un individuo después de tumbado o cortado. Se contabilizaron todos los árboles con un $1 \mathrm{~cm}$ de DAP y $1.30 \mathrm{~m}$ de altura en al menos uno de los tallos, que fueron entonces considerados como los individuos con rebrote en los cálculos posteriores. En campo es difícil identificar si un árbol con tallo único se originó por rebrote, por lo tanto, muchas especies que durante un inventario o muestreo no presentan esta característica, pueden quedar excluidas y erróneamente consideradas como incapaces de rebrotar. Para mejorar la comprensión de este fenómeno, se realizó una entrevista estructurada durante un recorrido de campo, en la cual se preguntaba a un parataxónomo local como informante clave, si las especies que no fueron reportadas en las parcelas de muestreo con rebrotes, eran capaces de rebrotar. En los casos en que no se obtuvo información de campo o por el informante, se realizó la revisión de literatura, sobre ese potencial. Con los datos recabados en campo, se calculó la proporción de especies con y sin rebrote para cada etapa en la que se dividió la sucesión para fines de este estudio.

Análisis estadísticos: En los análisis estadísticos solamente se incluyeron los datos recabados en las parcelas, excluyéndose especies para las cuales se obtuvo la información del informante clave o de revisión de literatura. Para el análisis de agrupamientos por similitud florística se utilizó el método de agrupamiento de pares promedio sin ponderación (UPGMA), determinando si existían diferencias en la composición de especies entre las etapas sucesionales por medio del análisis de similitud (ANOSIM), identificando especies indicadoras mediante el análisis SIMPER, con base en 
el aporte porcentual que cada especie da a la disimilitud entre pares de etapas comparadas. La medida de distancia utilizada en todas estas pruebas fue el índice Bray-Curtis. Para todos los análisis se utilizó el programa estadístico PAST 3.11 (Hammer, Harper, \& Ryan, 2001).

Establecida la normalidad en la distribución de los datos mediante la prueba de Kolmogorov-Smirnof ( $\mathrm{P}>0.05)$, y para conocer las diferencias en individuos con rebrote entre etapas, se realizó un análisis de varianza (ANDEVA) considerando $\mathrm{P}<0.05$ como significativo, posteriormente se aplicó un análisis de separación de medias con el método de Tukey (95\% de confianza), mediante el programa SPSS para ver si hay diferencias significativas entre grupos (Morrison, 1999).

\section{RESULTADOS}

Se registraron 13972 individuos $(32 \%$ con rebrote y $68 \%$ sin rebrote, Apéndice 1), pertenecientes a 168 especies (79\% con rebrote y 21 $\%$ sin rebrote) y 44 familias. De estas especies, 133 fueron observadas con rebrotes en campo en al menos una etapa sucesional. De las 35 especies que no presentaron esta característica en campo, 34 fueron reportadas con capacidad de rebrote por el informante clave, dando lugar al incremento hasta el $99.4 \%$ del total reportado en el área de estudio (Apéndice 1). Sólo para Bourreria mollis no se obtuvo información sobre esta capacidad.

La familia con mayor riqueza fue Fabaceae (32 especies), seguida por Rubiaceae (11),

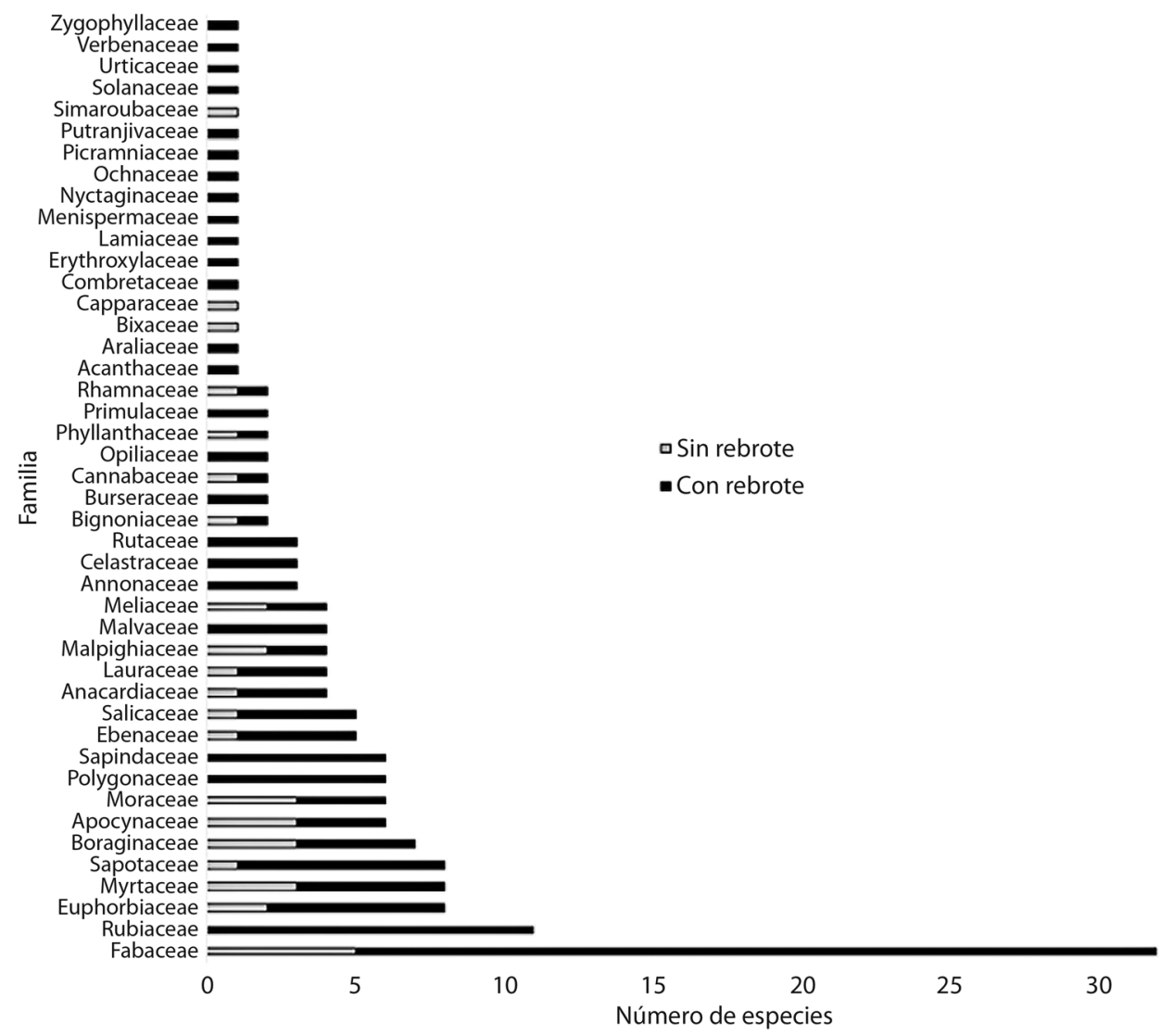

Fig. 2. Número de especies agrupadas por familia indicando cuales tuvieron o no rebrote, dentro del bosque tropical estudiado en Calakmul, Campeche, México.

Fig. 2. Species number clumped by family indicating which have or not sprouting, inside the tropical forest studied in Calakmul, Campeche, Mexico. 
Euphorbiaceae, Myrtaceae, Sapotaceae (8), Boraginaceae (7), Apocynaceae y Moraceae (6), que en conjunto aportan más del $50 \%$ de la riqueza total (Fig. 2). Al analizar la proporción del rebrote entre las diferentes etapas, la familia Fabaceae predomina con 27 especies con rebrote contra cinco que no, para el caso de Rubiaceae todas las especies rebrotaron. Tres familias (Bixaceae, Capparaceae y Simaroubaceae) no se registraron en campo a sus especies con capacidad de rebrote. Todas las demás familias presentaron menos de ocho especies.

Al realizar el análisis de individuos con y $\sin$ rebrote, la familia Fabaceae ocupa la primera posición. Euphorbiaceae, Myrtaceae y Polygonaceae ocuparon respectivamente el segundo, tercer y cuarto lugar (Fig. 3).

El rebrote está presente en toda la sucesión, tendiendo a disminuir tanto en especies como en individuos que lo presentan en función de la edad (Fig. 4 y Fig. 5). El número de individuos con rebrote fue significativamente diferente entre etapas sucesionales (ANDEVA, $F=5.725, P=0.003)$. Según la prueba de Tukey, los promedios en el número de rebrotes que presentan los bosques secundarios, BSJ $(1189 \pm 537 \mathrm{ind} / \mathrm{ha})$, BSI (1 $408 \pm 729 \mathrm{ind} / \mathrm{ha})$, BSA (1 $248 \pm 597 \mathrm{ind} / \mathrm{ha})$, forman una categoría similar entre sí, que es superior al promedio de BT (235 \pm 152 ind/ha). Lo anterior se corresponde con los resultados que se obtienen para el análisis UPGMA (Fig. 6), que muestra como los bosques maduros (BT) conforman un grupo distinto al de los bosques secundarios (BSJ, BSI y BSA), el mismo análisis muestra que dentro de cada etapa hay una alta heterogeneidad o disimilitud. La figura 4 y la figura 5 explican el valor porcentual de especies e individuos, respectivamente para cada etapa. Al realizar el análisis UPGMA para todas las especies se observó que la similitud es menor a $30 \%$ (Fig. 6).

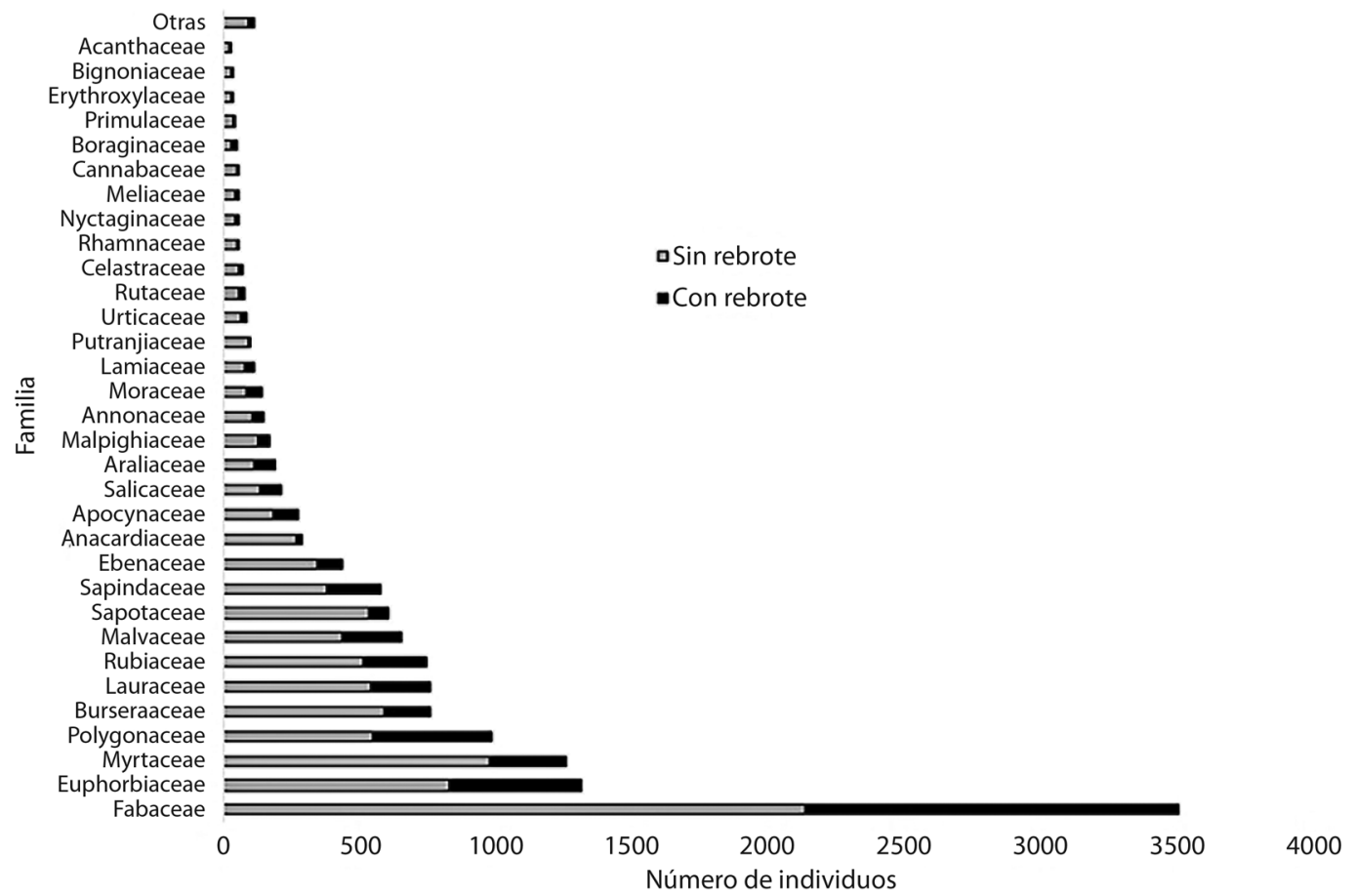

Fig. 3. Número de individuos agrupados por familia indicando el número cuales tuvieron o no rebrote, en el bosque tropical estudiado en Calakmul, Campeche, México.

Fig. 3. Individual number clumped by family indicating which have or not sprouting, inside the tropical forest studied in Calakmul, Campeche, Mexico. 


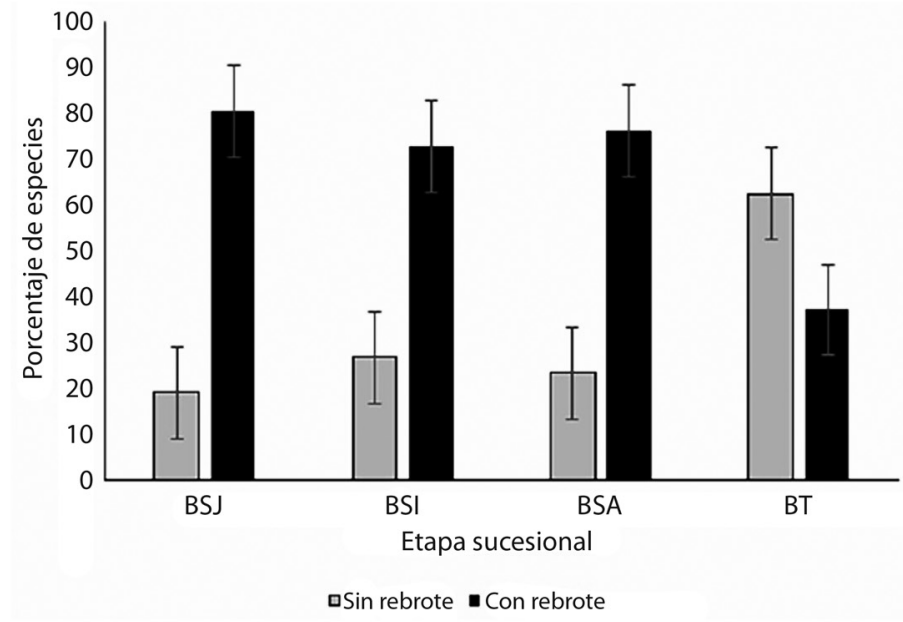

Fig. 4. Porcentaje de especies con y sin rebrote en cuatro etapas sucesionales en el bosque tropical de Calakmul, Campeche, México. Códigos: Bosque secundario Joven (BSJ), intermedio (BSI) y avanzado (BSA), bosque conservado (BT).

Fig. 4. Percentage of species with and without sprouts in four successional stages in the tropical forest of Calakmul, Campeche, Mexico. Codes: Young Secondary forest (BSJ), intermediate (BSI) and Advanced (BSA), preserved tropical forest (BT).

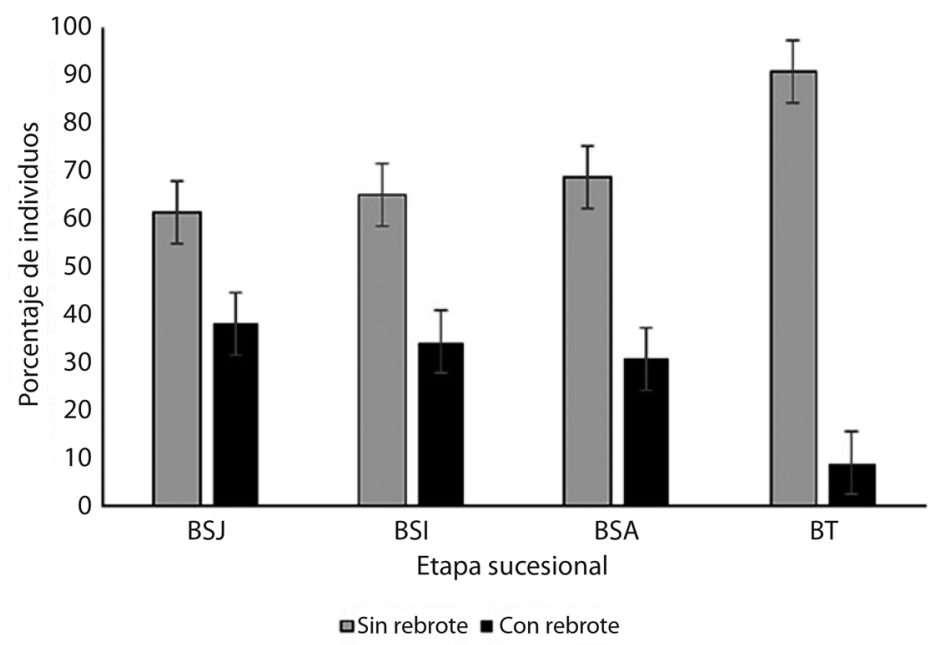

Fig. 5. Porcentaje de individuos con y sin rebrote en cuatro etapas sucesionales en el bosque tropical de Calakmul, Campeche, México. Códigos: Bosque secundario Joven (BSJ), intermedio (BSI) y avanzado (BSA), bosque tropical conservado (BT). Fig. 5. Percentage of individuals with and without sprouts in four successional stages in the tropical forest of Calakmul, Campeche, Mexico. Codes: Young Secondary forest (BSJ), intermediate (BSI) and Advanced (BSA), preserved tropical forest (BT).

El análisis de similitud (ANOSIM) mostró que la composición de especies entre las etapas en la sucesión, fue estadísticamente diferente $(\mathrm{R}=0.2634, \mathrm{P}=0.0001)$. Se verifica esto al comparar entre estos mediante el análisis de similitud porcentual o SIMPER, detectándose especies que aportan mucho a la diferenciación sucesional (Cuadro 2) y que además son capaces de rebrotar. Relacionado a lo anterior, los análisis de similitud y de especies indicadoras muestran que cada etapa tiene especies que la distinguen del bosque tropical, como 


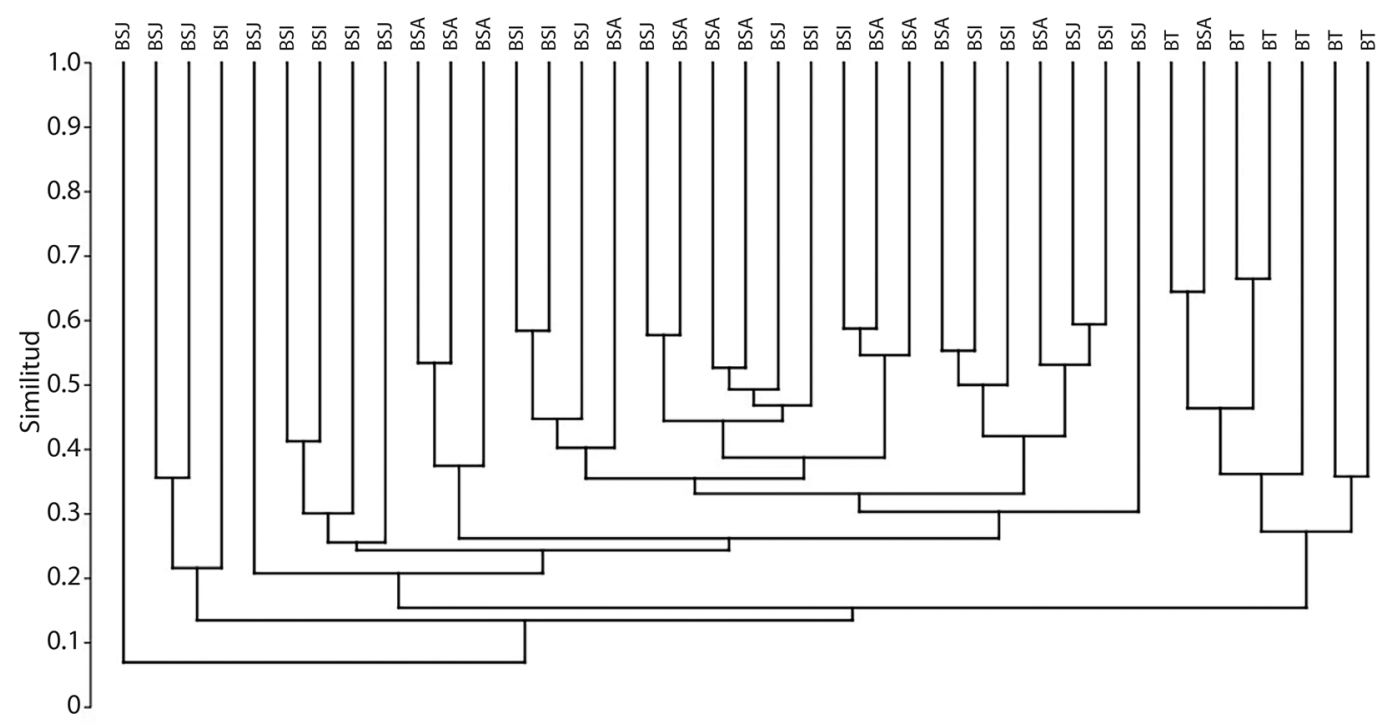

Fig. 6. Análisis UPGMA que muestra la heterogénea composición de especies en cuatro etapas sucesionales del bosque tropical de la Reserva de la Biósfera de Calakmul, Campeche, México. Para convertir a porcentaje, el valor escalar se multiplica por 10. Bosque secundario Joven (BSJ), intermedio (BSI) y avanzado (BSA), bosque conservado (BT).

Fig. 6. UPGMA analysis showing the heterogeneous species composition in four sucesional stages in the tropical forest from Calakmul, Biosphere Reserve, Campeche, Mexico. To convert so percentage, 10 multiply the scalar value. Codes: Young Secondary forest (BSJ), intermediate (BSI) and Advanced (BSA), preserved tropical forest (BT).

CUADRO 2

Especies que contribuyen con mayor porcentaje de la disimilitud según el análisis SIMPER.

Códigos: Bosque secundario Joven (BSJ), intermedio (BSI) y avanzado (BSA), bosque tropical conservado (BT)

TABLE 2

Species that contribute the highest percentage of dissimilarity according to the SIMPER analysis. Codes: Young Secondary forest (BSJ), intermediate (BSI) and Advanced (BSA), preserved tropical forest (BT)

\begin{tabular}{lccc}
\multicolumn{1}{c}{ Especie } & BT-BSJ & & \\
Lonchocarpus xuul & Disimilitud (promedio) & \% Contribución & \% Acumulativo \\
Hampea trilobata & 4.93 & 5.52 & 13.73 \\
Piscidia piscipula & 4.56 & 5.11 & 18.84 \\
Bursera simaruba & 4.27 & 4.78 & 23.62 \\
Nectandra salicifolia & 3.00 & 3.36 & 35.42 \\
Lonchocarpus rugosus & 3.89 & 4.35 & 27.97 \\
Diospyros salicifolia & 1.70 & 1.90 & 48.47 \\
Thevetia ahouai & 1.89 & 2.11 & 42.52 \\
\hline
\end{tabular}


CUADRO 2 (Continuación) / TABLE 2 (Continued)

\begin{tabular}{|c|c|c|c|}
\hline \multicolumn{4}{|c|}{ BT-BSI } \\
\hline Especie & Disimilitud (promedio) & \% Contribución & $\%$ Acumulativo \\
\hline Lonchocarpus xuul & 8.57 & 9.97 & 9.97 \\
\hline Croton icche & 3.80 & 4.42 & 21.75 \\
\hline Eugenia ibarrae & 3.03 & 3.52 & 37.40 \\
\hline Bursera simaruba & 3.47 & 4.03 & 30.15 \\
\hline Nectandra salicifolia & 3.76 & 4.37 & 26.12 \\
\hline Guettarda combsii & 2.54 & 2.95 & 40.35 \\
\hline Coccoloba reflexiflora & 2.09 & 2.43 & 48.10 \\
\hline Hampea trilobata & 2.31 & 2.69 & 43.04 \\
\hline Croton arboreus & 1.93 & 2.24 & 52.59 \\
\hline Dendropanax arboreus & 1.94 & 2.25 & 50.35 \\
\hline \multicolumn{4}{|c|}{ BT-BSA } \\
\hline Especie & Disimilitud (promedio) & \% Contribución & $\%$ Acumulativo \\
\hline Lonchocarpus xuul & 6.00 & 7.42 & 15.17 \\
\hline Eugenia ibarrae & 4.04 & 5.00 & 20.16 \\
\hline Nectandra salicifolia & 3.36 & 4.16 & 28.71 \\
\hline Bursera simaruba & 2.99 & 3.70 & 32.40 \\
\hline Croton arboreus & 2.01 & 2.48 & 37.38 \\
\hline Neomillspaughia emarginata & 1.99 & 2.46 & 39.85 \\
\hline Piscidia piscipula & 1.87 & 2.31 & 44.61 \\
\hline Lonchocarpus yucatanensis & 2.02 & 2.50 & 34.90 \\
\hline Hampea trilobata & 1.68 & 2.07 & 48.78 \\
\hline Coccoloba reflexiflora & 1.57 & 1.94 & 52.68 \\
\hline Diospyros salicifolia & 1.54 & 1.90 & 54.58 \\
\hline
\end{tabular}

su referente ecológico de vegetación madura (BT). De esta manera Lonchocarpus xuul, Hampea trilobata y Bursera simaruba son las especies que más aportan a distinguir un bosque secundario (BSJ, BSI o BSA) de un BT, por ser más abundantes en la etapa joven de la sucesión. Lo contrario ocurre respecto a Nectandra salicifolia, Pouteria reticulata, Gymnanthes lucida, Drypetes lateriflora, Manilkara zapota y Mosannona depressa que se encontraron en mayor número dentro del BT, por lo que son indicadoras de un bosque conservado o antiguo en esa zona (Cuadro 2).

\section{DISCUSIÓN}

El número de especies que presentaron rebrotes en parcelas de muestreo en campo subestimó la verdadera dimensión de este atributo para el bosque tropical en Calakmul, donde se encuentra una proporción de 133 especies con rebrote de 168 en total, lo que se evidenció al obtenerse información mediante el informante clave, quien aportó datos que permitieron elevar hasta el 167 el número de especies que pueden reiniciar su crecimiento a partir de brotes surgidos de tallo o raíz (99.4\% del total). Así, de las 35 especies que inicialmente se reportan como sin rebrotes en campo, solamente una, Bourreria mollis, queda sin información sobre esta capacidad desde revisión de literatura, aunque el informante clave menciona que esta especie no lo hace. Su aporte al conjunto del arbolado es poco influyente en los resultados estadísticos, debido a que, siguiendo la definición de Halftter y Escurra (1992), es una especie demográficamente rara en el bosque maduro. Con base en los datos de campo y los complementarios datos aportados por el informante clave y la revisión de literatura, se puede 
afirmar que la mayoría de los árboles de Calakmul pueden regenerarse a partir de tocones. Una situación semejante reportan Boucher et al. (2001) quienes al estudiar el proceso de regeneración del bosque tropical después de un daño por huracán, concluyen que más del 70 $\%$ de las especies resurgen de rebrotes. Islebe et al. (2009) menciona algo similar respecto al bosque de mangle en Quintana Roo, donde menciona que después del paso del huracán Dean, la re-foliación fue de un $80 \%$.

La capacidad de rebrote puede variar entre las especies y familias botánicas, y por lo tanto, puede ser un factor importante en la aptitud competitiva de las mismas (Bellingham \& Sparrow, 2000; Toledo-Araneda, 2005). Al respecto, la familia Fabaceae, que cuenta con numerosas especies forrajeras, capaces de rebrotar después de poda (Salgado \& SilvaZamora, 2008) y resistentes al fuego (ChanDzul, 2010), destaca como la más importante en todas las etapas sucesionales. La predominancia de esta familia es un carácter distintivo de los bosques de la Península de Yucatán (Chan-Dzul, 2010; Duno De Estefano \& Cetzal-Ix, 2016; Zamora-Crescencio et al., 2017) y Tabasco (González-Valdivia, Cetzal-Ix, \& Dzib-Castillo, 2016). Esta situación permite que algunas especies se constituyan como indicadoras de una condición del bosque en sucesión y lo distinga del estado más estable o maduro del ecosistema. Así Lonchocarpus xuul y otras leguminosas pueden ser más fácilmente encontradas en vegetación secundaria que en un bosque maduro en Calakmul, tal como mencionan Báez-Vargas et al. (2017).

El porcentaje de individuos con rebrote va disminuyendo conforme avanza la edad de la sucesión, lo que coincide con lo señalado por Kammesheidt (1998) quien encontró que el mayor número de individuos con rebrotes se encuentra en el bosque secundario joven. Martínez-Ramos y García-Orth (2007), señalan que los bosques del neotrópico son capaces de resistir a la roza-tumba y quema, recuperándose en tiempo cortos de barbecho. Aunque este proceso de disminución de número de individuos y especies con rebrotes conforme se incrementa el tiempo del barbecho ha sido registrado, puede subestimar la realidad, debido a procesos relacionados como la auto poda (Toledo-Araneda, 2005) o la dominancia apical (Salgado \& Silva-Zamora, 2008). Esto puede conllevar a que un árbol de múltiples tallos pierda varios tallos en función del tiempo, dejando solamente el tallo dominante, perdiéndose así la evidencia de la condición inicial conforme crece el árbol.

La combinación de técnicas de muestreo en parcelas establecidas en campo con la entrevista etnobotánica al informante clave, permitió ilustrar de mejor manera el fenómeno del rebrote en el arbolado en Calakmul, estableciendo que la mayoría de las especies arbóreas pueden, después de la roza-tumbaquema, crecer y recuperar su presencia, restituyendo la cobertura forestal y composición de las especies que lo caracterizan en pocas décadas. En estos ecosistemas la distinción entre la etapa secundaria con respecto al bosque conservado o maduro, se vuelve difícil, si se analizan variables estructurales como DAP, áreas basales o altura del arbolado, más si se comparan etapas avanzadas de sucesión, cercanas a los 50 años de edad en barbecho, con los bosques de referencia ( $>100$ años sin evidencia de uso agropecuario) (Aryal et al., 2014). Debe considerarse que en la Península de Yucatán, los Mayas alcanzaron densidades de población superiores a las actuales (150 a $800 \mathrm{hab} / \mathrm{km}^{2}$ ) (Turner, 1987), sustentadas en el sistema de producción agroforestal rotacional de cultivos-bosques, mejor conocido como milpa maya, el cual es una fuerza de selección para especies resilientes (Ford \& Nigh, 2010; Mariaca-Méndez, 2015). Es deducible que el cambio de este tipo de práctica por otras que eliminen los tocones tendrá más impacto sobre la flora y la diversidad.

Los análisis realizados con respecto a la composición de especies, muestran que, en Calakmul, los bosques maduros entre sí tienden a ser intrínsecamente diferentes en sus asociaciones arbóreas y entonces se muestran heterogéneos entre sitios. Los bosques secundarios derivados de ellos, en consecuencia, 
también difieren entre sí. Las diferencias entre el bosque secundario y el bosque maduro superan $70 \%$, pero estas son altas incluso dentro de unidades de la misma etapa sucesional (BáezVargas et al., 2017). El patrón de similitud entre los bosques secundarios y maduros que se presentan en esta región, ha sido reportado en otros estudios, por ejemplo González-Valdivia et al. (2012), al estudiar bosques tropicales en Tenosique, Tabasco, México, señalan que los bosques conservados con respecto a los secundarios la similitud del arbolado es menor que el $20 \%$. Las causas de la heterogeneidad interna en estos bosques, tanto primarios como sucesionales, no han sido plenamente dilucidadas, pero en el caso de Calakmul, posiblemente se relacionen con la abigarrada variedad de microcondiciones de suelo y relieve, la hidrología, a la exposición de las pendientes y a la actividad milenaria del hombre (Duno de Stefano et al., 2018), además de las variaciones y heterogeneidad ecosistémica, que resultan de los disturbios naturales frecuentes, como explican Swanson et al. (2010). Más investigaciones en este campo se hacen necesarias para comprender la sucesión (Dupuy et al., 2012).

En términos de aplicación de los resultados a fortalecer algún proceso de intervención en los bosques, sea por motivos de conservación o de aprovechamiento y manejo sustentable, los resultados pueden aportar a la distinción de bosques secundarios o llamados localmente "acahuales", de los bosques conservados en la Reserva de la Biósfera de Calakmul y sus áreas de amortiguamiento. Es decir, al notarse una disminución en el número de individuos con múltiples tallos en las unidades de muestreo establecidas en los bosques maduros, en contraste con lo que ocurre conforme la sucesión está en una etapa más temprana, puede emplearse la presencia de numerosos árboles con rebrotes como evidencia de que se está en un bosque en fase de regeneración temprana y entonces legislar con base en estos hechos y otros aspectos bien documentados de la estructura y composición de estos ecosistemas (Aryal et al., 2014; García-Licona et al., 2014; Baéz-Vargas et al., 2017) una mejor propuesta de manejo, como sugieren Román-Dañobeytia et al. (2014).

Los datos obtenidos y los resultados de los análisis realizados a partir de ellos, evidencias que, en términos generales, prácticamente todas las especies arbóreas en las etapas sucesionales del bosque estudiado en Calakmul, presentan la capacidad de rebrotar, lo cual tiene influencia en el rápido proceso de recuperación del bosque después de la roza-tumba y quema. Si se cambia este sistema por otros más intensivos o que limitan la cantidad de tocones que se dejan al utilizar un campo para cultivo o ganado, se anula la capacidad de regeneración natural de estos ecosistemas, y se elimina el principal atributo de su resiliencia ecológica.

Declaración de ética: los autores declaran que todos están de acuerdo con esta publicación y que han hecho aportes que justifican su autoría; que no hay conflicto de interés de cualquier tipo; y que han cumplido con todos los requisitos y procedimientos éticos y legales pertinentes. El documento firmado se encuentra en los archivos de la revista.

\section{AGRADECIMIENTOS}

A Demetrio Álvarez Montejo por el apoyo en la identificación de las especies arbóreas. A los ejidatarios de los ejidos Cristóbal Colón, El Carmen II, Narciso Mendoza y Nuevo Conhuás, de la región de Calakmul, Campeche, México por habernos permitido el acceso a sus parcelas. Al Consejo Nacional de Ciencia y Tecnología (CONACyT), por la beca no. 598724 otorgada a la primera autora. A El Colegio de la Frontera Sur por apoyar la ejecución en campo de este estudio. A cinco revisores anónimos que ayudaron a enriquecer el documento.

\section{RESUMEN}

Los estudios detallados para comprender como los bosques neotropicales se reestablecen en el tiempo, a través de la sucesión secundaria aún resultan necesarios. Entre los mecanismos de regeneración que actúan en los bosques 
tropicales, el rebrote de árboles predomina en especies leñosas, con la ventaja de reiniciar el crecimiento de raíces establecidas, que permiten restituir el dosel forestal. El objetivo de este estudio fue identificar y cuantificar las especies arbóreas con capacidad de rebrote en distintas etapas de la sucesión secundaria (barbecho) después del sistema agrícola tradicional de milpa, comparadas con las comunidades del bosque tropical predominante en la Reserva de la Biósfera de Calakmul, y cómo influyen estas especies en la recuperación de la composición de este ecosistema. Se calculó el porcentaje de especies con y sin rebrote para cada etapa sucesional. Se registraron 13972 individuos (32\% con rebrote y $68 \%$ sin rebrote) y 168 especies (79\% con rebrote y $21 \%$ sin rebrote), el porcentaje de individuos con rebrote va disminuyendo conforme avanza la edad de la sucesión. El número de individuos con rebrote y la composición de especies fueron significativamente diferentes entre etapas. Se puede concluir que la mayoría de especies arbóreas del bosque en Calakmul, presentan la capacidad de rebrotar, lo cual tiene influencia en el rápido proceso sucesional del bosque después de la roza-tumba y quema, restituyendo la cobertura y estructura forestal, así como la composición de las especies originales.

Palabras clave: sucesión secundaria; especie arbórea; rebrote; composición de especies; bosque tropical.

\section{REFERENCIAS}

Aryal, D. R., de Jong, B. H. J., Ochoa-Gaona, S., EsparzaOlguín, L., \& Mendoza-Vega, J. (2014). Carbon stocks and changes in tropical secondary forests of southern Mexico. Agriculture, Ecosystems and Environment, 195, 220-230.

Báez-Vargas, A. M., Esparza-Olguín, L., Martínez-Romero, E., Ochoa-Gaona, S., Ramírez-Marcial, N., \& González-Valdivia, N. A. (2017). Efecto del manejo sobre la diversidad de árboles en vegetación secundaria en la Reserva de la Biosfera de Calakmul, Campeche, México. Revista de Biología Tropical, 65(1), 41-53.

Baider, C., Tabarelli, M., \& Mantovani, W. (2001). The soil seed bank during Atlantic forest regeneration in Southeast Brazil. Revista Brasileira de Biologia, 61(1), 35-44.

Barbosa, J. M., Broadbent, E. N., \& Bitencourt, M. D. (2014). Remote sensing of aboveground biomass in tropical secondary forests: a Review. International Journal of Forestry Research, 2014, 1-14.

Bellingham, P. J., \& Sparrow, A. D. (2000). Resprouting as a life history strategy in woody plant communities. OIKOS, 89(2), 409-416.

Benítez-Malvido, J., Martínez-Ramos, M., \& Ceccon, E. (2001). Seed rain vs. seed bank, ant the effect of vegetation cover on the recruitment of tree seedlings in tropical successional vegetation. Dissertation Botanicae, 346, 185-203.

Boucher, D. H., Vandermeer, J. H., Granzow de la Cerda, I., Mallona, M. A., Perfecto, I., \& Zamora, N. (2001). Post-Agriculture versus Post-Hurricane Succession in Southeastern Nicaraguan Rain Forest. Plant Ecology, 156(2), 131-137.

Brown, S., \& Lugo, A. E. (1990). Tropical secondary forests. Journal of Tropical Ecology, 6(1), 1-32.

Carrillo-Bastos, A., Elizalde-Rendón, E. M., TorrescanoValle, N., \& Flores-Ortiz, G. (2008). Adaptación ante disturbios naturales, manglar de Puerto Morelos, Quintana Roo, México. Foresta Veracruzana, 10(1), 31-38.

Chan-Dzul, A. (2010). Diversidad florística y funcional a través de una cronosecuencia de la selva mediana subperennifolia en la zona de influencia de la Reserva de la Biósfera Calakmul, Campeche, México (Tesis de Maestría). Centro Agronómico Tropical de Investigación y Enseñanza (CATIE), Costa Rica.

Chazdon, R. L., Peres, C. A., Dent, D., Sheil, D., Lugo, A. E., Lamb, D., ... Miller, S. E. (2009). The potential for species conservation in tropical secondary forests. Conservation Biology, 23(6), 1406-1417.

Duno de Estefano, R. \& Cetzal-Ix, W. (2016). Fabaceae (Leguminosae) en la Península de Yucatán, México. Desde el Herbario CICY, 8, 111-116.

Duno de Stefano, R., Ramírez-Morillo, I., Tapia-Muñoz, J. L., Hernández-Aguilar, S., Can, L. L., Cetzal-Ix, W., ... Fernández-Concha, G. (2018). Aspectos generales de la flora vascular de la Península de Yucatán Mexicana. Botanical Sciences (Online First), 383-400. DOI: $10.17129 /$ botsci.1868

Dupuy, J. M., Hernández-Stefanoni, J. L., HernándezJuárez, R. A., Tetetla-Rangel, E., López-Martínez, J. O., Leyequién-Abarca, E., ... May-Pat, F. (2012). Patterns and correlates of tropical dry forest structure and composition in a highly replicated chronosequence in Yucatán, Mexico. Biotropica, 44, 151-162.

Dzib-Castillo, B. B., Chanatásig-Vaca, C., \& GonzálezValdivia, N. A. (2014). Estructura y composición en dos comunidades arbóreas de la selva baja caducifolia y mediana subcaducifolia en Campeche, México. Revista Mexicana de Biodiversidad, 85(1), 167-178.

Fahey, R. T., \& Puettmann, K. (2007). Ground layer disturbance and initial conditions influence gap portioning of understory vegetation. Journal of Ecology, 95(5), 1098-1109.

Ferguson, B. G., \& Grifttin, D. M. (2004). Tecnología agrícola y conservación biológica en el Peten, Guatemala. Manejo Integrado de Plagas y Agroecología (Costa Rica), 72, 72-85. 
Ford, A., \& Nigh, R. (2010). The Milpa cycle and the making of the Maya Forest garden. In J. Morris, S. Jones, J. Awe, G. Thompson, \& M. Badillo (Eds.), Archaeological Investigations in the Eastern Maya Lowlands (pp. 183-190). Belmopan, Belize: Institute of Archaeology.

García-Licona, J. B., Esparza-Olguín, L. G., \& MartínezRomero, E. (2014). Estructura y composición de la vegetación leñosa de selvas en diferentes estadios sucesionales en el ejido El Carmen II, Calakmul, México. Polibotanica, 38, 1-26.

Gómez-Pompa, A., \& Vázquez-Yanes, C. (1985). Estudios sobre la regeneración de selvas en regiones cálidohúmedas de México. En A. Gómez-Pompa \& R. S. Del Amo (Eds.), Investigaciones sobre la Regeneración de Selvas Altas en Veracruz, México (pp. 1-25). Xalapa, Veracruz: Instituto Nacional de Investigaciones sobre Recursos Bióticos.

González-Iturbe, J. A., Olmsted, I., \& Tun-Dzul, F. (2002). Tropical dry forest recovery after long term Henequen (sisal, Agave fourcroydes Lem.) plantation in northern Yucatan, Mexico. Forest Ecology and Management, 167, 67-82.

González-Valdivia, N. A., Cetzal-Ix, W., \& Dzib-Castillo, B. (2016). Leguminosas en un paisaje de la sierra de Tenosique, Tabasco, México. Desde el Herbario CICY, 8, 27-29.

González-Valdivia, N., Ochoa-Gaona, S., Ferguson, B. G., Pozo, C., Kampichler, C., \& Pérez-Hernández, I. (2012). Análisis comparativo de la estructura, diversidad y composición de comunidades arbóreas de un paisaje agropecuario en Tabasco, México. Revista Mexicana de Biodiversidad, 83(1), 83-99.

Granda-Moser, V., Finegan, B., Ramos-Bendaña, Z. S., Detlefsen, G., \& Molina, A. (2015). Potencial de manejo de bosques restaurados por sucesión natural secundaria en Guanacaste, Costa Rica: Composición, diversidad y especies maderables. Turrialba, Costa Rica: Centro Agronómico Tropical de Investigación y Enseñanza (CATIE).

Guariguata, M. R., \& Ostertag, R. (2001). Neotropical secondary forest succession: changes in structural and functional characteristics. Forest Ecology and Management, 148, 185-206.

Halftter, G. \& Escurra, E. (1992). ¿Qué es la biodiversidad? En G. Halffter (Comp.), La diversidad biológica de Iberoamérica I (pp. 3-24). Veracruz, México: Instituto de Ecología A.C.

Hammer, Ø., Harper, D. A. T., \& Ryan, P. D. (2001). PAST: Paleontological statistics software package for education and data analysis. Paleontología Electrónica, 4, 1-9.
INE (2000). Programa de Manejo de la Reserva de la Biósfera de Calakmul. México D.F.: Instituto Nacional de Ecología (INE).

Islebe, G. A., Torrescano-Valle, N., Valdez-Hernández, M., Tuz-Novelo, M., \& Weissenberger, H. (2009). Efectos del impacto del huracán Dean en la vegetación del sureste de Quintana Roo, México. Foresta Veracruzana, 11(1), 1-6.

ITTO. (2002). ITTO Guidelines for the restoration, management and rehabilitation of degraded and secondary tropical forests. Yokohama, Japan: International Tropical Timber Organization (ITTO).

Kammesheidt, L. (1998). The role of tree sprouts in the restoration of stand structure and species diversity in Tropical Moist Forest after slash-and-burn agriculture in Eastern Paraguay. Plant Ecology, 139(2), 155-165.

Levy-Tacher, S. \& Hernández-Xolocotzi, E. (1992). La sucesión secundaria en Yucatán y su manejo. En D. Zizumbo-Villareal, C. H. Rasmussen, M. AriasReyes, \& S. Terán-Contreras (Eds.), La modernización de la milpa en Yucatán: utopía o realidad (pp. 203-214). Yucatán, México: Centro de Investigación Científica de Yucatán (CICY).

Mariaca-Méndez, R. (2015). La milpa maya yucateca en el Siglo XVI: evidencias etnohistóricas y conjeturas. Etnobiología, 13(1), 1-38.

Martínez-Ramos, M. \& García-Orth, X. (2007). Sucesión ecológica y restauración de las selvas húmedas. Boletín de la Sociedad Botánica de México, 80, 69-84.

Martínez-Salas, E. \& Galindo-Leal, C. (2002). La vegetación de Calakmul, Campeche, México: Clasificación, Descripción y Distribución. Boletín de la Sociedad Botánica de México, 71, 10-19.

Morrison, E. (1999). Introduction to SPSS. Washington D.C., EE.UU.: Center for Social Science Computation and Research, Universidad de Washington. Recuperado de http://julius.csscr.washington.edu/ pdf/spss_old.pdf

Ochoa-Gaona, S., \& González-Espinosa, M. (2000). Land use and deforestation in the highlands of Chiapas, Mexico. Applied Geography, 20(1), 17-42.

Ochoa-Gaona, S., Hernández-Vásquez, F., de Jong, B. H. J., \& Gurri-García, F. D. (2007). Pérdida de diversidad florística ante un gradiente de intensificación del sistema agrícola de roza-tumba-quema: Un estudio de caso en la selva Lacandona, Chiapas, México. Boletín de la Sociedad Botánica de México, 81, 65-80.

Paciorek, C. J., Condit, R., Hubbell, S. P., \& Foster, R. B. (2000). The demographics of resprouting in tree and shrub species of a moist tropical forest. Journal of Ecology, 88(5), 765-777. 
Pennington, T., \& Sarukhán, J. (2005). Árboles tropicales de México. Manual para la identificación de las principales especies. México, D.F.: Fondo de Cultura Económica.

Poorter, L., Bongers, F. M., Aide, T. M., Almeyda Zambrano, A. M., Balvanera, P., Becknell, J. M., ..., Rozendaal, D. M. A. (2016). Biomass resilience of Neotropical secondary forests. Nature, 530, 211-214.

Poorter, L., Kitajima, K., Mercado, P., Chubiña, J., Melgar, I., \& Prins, H. H. T. (2010). Resprouting as a persistence strategy of tropical forest trees: relations with carbohydrate storage and shade tolerance. Ecology, 91(9), 2613-2627.

Quesada, M., Sanchez-Azofeifa, A., Alvarez-Añorve, M., Stoner, K. E., Avila-Cabadilla, L., Calvo-Alvarado, J., ... Sánchez-Montoya, G. (2009). Succession and management of tropical dry forests in the Americas: review and new perspectives. Forest Ecology and Management, 258(6), 1014-1024.

Rico-Gray, V., García-Franco, J. G., Puch, A., \& Simá, P. (1988). Composition and structure of a tropical dry forest in Yucatán, Mexico. International Journal of Ecology and Environmental Science, 14, 21-29.

Román-Dañobeytia, F. J., Levy-Tacher, S. I., MacarioMendoza, P., \& Zúñiga-Morales, J. (2014). Redefining Secondary Forests in the Mexican Forest Code: Implications for Management, Restoration, and Conservation. Forest, 5, 978-991. DOI:10.3390/f5050978

Salgado, O. D. \& Silva-Zamora, C. E. (2008). Evaluación de la capacidad de rebrote de dos especies arbóreas del Bosque Seco Secundario de Nandarola, Nandaime, Granada (Tesis de pregrado). Universidad Nacional Agraria, Managua, Nicaragua.

Swanson, M. E., Franklin, J. F., Beschta, R. L., Crisafulli, C. M., DellaSala, D. A., Hutto, R. L., ... \& Swanson, F. J. (2010). The forgotten stage of forest succession: early-successional ecosystems on forest sites.
Frontiers in Ecology and the Environment, 9(2), 117125. DOI: $10.1890 / 090157$

The Plant List. (2013). A Working List of All Plant Species (Versión 1.1.). Recuperado de http://www.theplantlist.org

Toledo-Araneda, L. A. (2005). Descripción del rebrote en cepas de Sequoia sempervirens (D.Don) Endl. (Tesis de pregrado). Universidad de Chile, Santiago, Chile.

Tropicos.org. (2018). Missouri Botanical Garden. Recuperado de http://www.tropicos.org

Turner, B. L. (1987). The Development and Demise of the Swidden Thesis of Maya Agriculture. In P. D. Harrison \& B. L. Turner (Eds.), Pre-Hispanic Maya Agriculture (pp. 13-22). Albuquerque: University of New Mexico Press.

Uhl, C. (1987). Factors controlling succession following slash and burn agriculture in Amazonia. Journal of Ecology, 75(29), 377-407.

Yih, K., Boucher, D. H., Vandermeer, J. H., \& Zamora, N. (1991). Recovery of the rain forest of Southeastern Nicaragua after destruction by hurricane Joan. Biotropica, 23(2), 106-113.

Zamora-Crescencio, P., Domínguez-Carrasco, M. R., Villegas, P., Gutiérrez-Báez, C., Manzanero-Acevedo, L. A., Ortega-Haas, J. J., ... Puch-Chávez, R. (2011). Composición florística y composición de la vegetación secundaria en el norte de Campeche, México. Boletín de la Sociedad Botánica de México, 89, 27-35.

Zamora-Crescencio, P., Rico-Gray, V., Barrientos-Medina, R. C., Puc-Garrido, E. C., Villegas, P., DomínguezCarrasco, M. del R., \& Gutiérrez-Báez, C. (2017). Estructura y composición florística de la selva mediana subperennifolia en Bethania, Campeche, México. Polibotánica, 43, 67-86. 


\section{APÉNDICE 1}

Listado de especies con y sin rebrote para las diferentes etapas sucesionales. BSJ: Bosque secundario joven, BSI: Bosque secundario intermedio, BSA: Bosque secundario avanzado, BT: Bosque tropical conservado

\section{APPENDIX 1}

List of species with and without sprouts for the different successional stages. BSJ: Young secondary forest, BSI: Intermediate secondary forest, BSA: Advanced secondary forest, BT: Preserved tropical forest

\begin{tabular}{|c|c|c|c|c|c|c|c|c|c|}
\hline \multirow[b]{2}{*}{ Familia } & \multirow[b]{2}{*}{ Especie } & \multicolumn{2}{|c|}{ BSJ } & \multicolumn{2}{|c|}{ BSI } & \multicolumn{2}{|c|}{ BSA } & \multicolumn{2}{|c|}{ BT } \\
\hline & & $\begin{array}{c}\text { Sin } \\
\text { rebrote }\end{array}$ & $\begin{array}{l}\text { Con } \\
\text { rebrote }\end{array}$ & $\begin{array}{l}\text { Sin } \\
\text { rebrote }\end{array}$ & $\begin{array}{l}\text { Con } \\
\text { rebrote }\end{array}$ & $\begin{array}{c}\text { Sin } \\
\text { rebrote }\end{array}$ & $\begin{array}{l}\text { Con } \\
\text { rebrote }\end{array}$ & $\begin{array}{c}\text { Sin } \\
\text { rebrote }\end{array}$ & $\begin{array}{l}\text { Con } \\
\text { rebrote }\end{array}$ \\
\hline Acanthaceae & Bravaisia berlandieriana & 0 & 0 & 0 & 0 & 0 & 0 & 19 & 4 \\
\hline Anacardiaceae & Astronium graveolens & 23 & 4 & 2 & 0 & 1 & 1 & 0 & 0 \\
\hline Anacardiaceae & Metopium brownei & 22 & 3 & 105 & 4 & 57 & 4 & 6 & 0 \\
\hline Anacardiaceae & Spondias mombin & 22 & 3 & 13 & 0 & 11 & 1 & 2 & 0 \\
\hline Anacardiaceae & Spondias sp. & 1 & 0 & 0 & 0 & 0 & 0 & 0 & 0 \\
\hline Annonaceae & Annona primigenia & 1 & 1 & 0 & 0 & 0 & 0 & 0 & 0 \\
\hline Annonaceae & Mosannona depressa & 7 & 2 & 26 & 18 & 35 & 14 & 34 & 5 \\
\hline Annonaceae & Sapranthus campechianus & 0 & 1 & 0 & 0 & 0 & 0 & 0 & 0 \\
\hline Apocynaceae & Aspidosperma megalocarpon & 0 & 0 & 0 & 0 & 0 & 0 & 3 & 1 \\
\hline Apocynaceae & Cameraria latifolia & 0 & 0 & 0 & 0 & 1 & 0 & 0 & 0 \\
\hline Apocynaceae & Cascabela gaumeri & 8 & 21 & 28 & 14 & 52 & 27 & 1 & 0 \\
\hline Apocynaceae & Plumeria obtusa & 0 & 0 & 0 & 0 & 0 & 0 & 2 & 0 \\
\hline Apocynaceae & Tabernaemontana litoralis & 2 & 0 & 0 & 0 & 0 & 0 & 0 & 0 \\
\hline Apocynaceae & Thevetia ahouai & 77 & 25 & 2 & 0 & 6 & 2 & 1 & 0 \\
\hline Araliaceae & Dendropanax arboreus & 10 & 14 & 85 & 51 & 13 & 8 & 7 & 0 \\
\hline Bignoniaceae & Handroanthus chrysanthus & 12 & 4 & 0 & 0 & 5 & 2 & 7 & 1 \\
\hline Bignoniaceae & Parmentiera millspaughiana & 0 & 0 & 1 & 0 & 0 & 0 & 0 & 0 \\
\hline Bixaceae & Cochlospermum vitifolium & 0 & 0 & 0 & 0 & 1 & 0 & 0 & 0 \\
\hline Boraginaceae & Bourreria mollis* & 0 & 0 & 1 & 0 & 0 & 0 & 1 & 0 \\
\hline Boraginaceae & Bourreria pulchra & 11 & 3 & 3 & 2 & 1 & 2 & 0 & 0 \\
\hline Boraginaceae & Cordia alliodora & 0 & 0 & 1 & 0 & 2 & 1 & 0 & 0 \\
\hline Boraginaceae & Cordia dentata & 0 & 0 & 0 & 0 & 0 & 1 & 0 & 0 \\
\hline Boraginaceae & Cordia dodecandra & 0 & 0 & 0 & 0 & 1 & 0 & 2 & 0 \\
\hline Boraginaceae & Ehretia tinifolia & 1 & 3 & 2 & 3 & 3 & 1 & 0 & 0 \\
\hline Boraginaceae & Rochefortia lundellii & 1 & 0 & 0 & 0 & 1 & 0 & 0 & 0 \\
\hline Burseraceae & Bursera simaruba & 123 & 78 & 219 & 30 & 192 & 58 & 11 & 0 \\
\hline Burseraceae & Protium copal & 0 & 0 & 25 & 1 & 6 & 0 & 17 & 0 \\
\hline Cannabaceae & Celtis trinervia & 1 & 0 & 1 & 0 & 0 & 0 & 0 & 0 \\
\hline Cannabaceae & Trema micrantha & 43 & 7 & 1 & 0 & 0 & 0 & 0 & 0 \\
\hline Capparaceae & Capparis indica & 1 & 0 & 0 & 0 & 0 & 0 & 2 & 0 \\
\hline Celastraceae & Crossopetalum gaumeri & 1 & 0 & 4 & 5 & 2 & 1 & 1 & 0 \\
\hline Celastraceae & Maytenus schippii & 0 & 0 & 2 & 1 & 1 & 0 & 2 & 0 \\
\hline Celastraceae & Semialarium mexicanum & 5 & 1 & 17 & 3 & 14 & 0 & 8 & 1 \\
\hline Combretaceae & Bucida buceras & 0 & 0 & 3 & 1 & 0 & 0 & 0 & 0 \\
\hline Ebenaceae & Diospyros anisandra & 0 & 2 & 3 & 0 & 33 & 2 & 0 & 0 \\
\hline Ebenaceae & Diospyros bumelioides & 4 & 5 & 2 & 0 & 0 & 0 & 0 & 0 \\
\hline Ebenaceae & Diospyros campechiana & 0 & 0 & 4 & 0 & 0 & 0 & 0 & 0 \\
\hline Ebenaceae & Diospyros salicifolia & 49 & 53 & 117 & 16 & 119 & 15 & 12 & 0 \\
\hline Ebenaceae & Diospyros yatesiana & 0 & 2 & 0 & 0 & 0 & 0 & 0 & 0 \\
\hline Erythroxylaceae & Erythroxylum rotundifolium & 0 & 1 & 4 & 0 & 11 & 10 & 10 & 0 \\
\hline
\end{tabular}


APÉNDICE 1 (Continuación) / APPENDIX 1 (Continued)

\begin{tabular}{|c|c|c|c|c|c|c|c|c|c|}
\hline \multirow[b]{2}{*}{ Familia } & \multirow[b]{2}{*}{ Especie } & \multicolumn{2}{|c|}{ BSJ } & \multicolumn{2}{|c|}{ BSI } & \multicolumn{2}{|c|}{ BSA } & \multicolumn{2}{|c|}{$\mathrm{BT}$} \\
\hline & & $\begin{array}{c}\text { Sin } \\
\text { rebrote }\end{array}$ & $\begin{array}{l}\text { Con } \\
\text { rebrote }\end{array}$ & $\begin{array}{c}\text { Sin } \\
\text { rebrote }\end{array}$ & $\begin{array}{l}\text { Con } \\
\text { rebrote }\end{array}$ & $\begin{array}{c}\text { Sin } \\
\text { rebrote }\end{array}$ & $\begin{array}{l}\text { Con } \\
\text { rebrote }\end{array}$ & $\begin{array}{c}\text { Sin } \\
\text { rebrote }\end{array}$ & $\begin{array}{l}\text { Con } \\
\text { rebrote }\end{array}$ \\
\hline Euphorbiaceae & Adelia barbinervis & 0 & 0 & 0 & 0 & 3 & 0 & 0 & 0 \\
\hline Euphorbiaceae & Croton arboreus & 17 & 34 & 112 & 55 & 98 & 70 & 7 & 0 \\
\hline Euphorbiaceae & Croton glabellus & 1 & 10 & 1 & 1 & 74 & 53 & 3 & 2 \\
\hline Euphorbiaceae & Croton icche & 15 & 34 & 155 & 167 & 50 & 31 & 7 & 0 \\
\hline Euphorbiaceae & Croton oerstedianus & 4 & 4 & 4 & 2 & 0 & 0 & 14 & 2 \\
\hline Euphorbiaceae & Gymnanthes lucida & 0 & 1 & 2 & 2 & 86 & 4 & 121 & 5 \\
\hline Euphorbiaceae & Jatropha gaumeri & 1 & 3 & 8 & 2 & 9 & 4 & 3 & 1 \\
\hline Euphorbiaceae & Sebastiania adenophora & 0 & 0 & 0 & 0 & 22 & 0 & 10 & 0 \\
\hline Fabaceae & Acacia angustissima & 0 & 0 & 0 & 0 & 4 & 0 & 0 & 0 \\
\hline Fabaceae & Acacia centralis & 5 & 1 & 7 & 0 & 3 & 2 & 4 & 0 \\
\hline Fabaceae & Acacia cornígera & 3 & 0 & 4 & 0 & 5 & 0 & 1 & 0 \\
\hline Fabaceae & Acacia dolichostachya & 4 & 1 & 10 & 0 & 14 & 1 & 0 & 0 \\
\hline Fabaceae & Acacia gaumeri & 4 & 12 & 1 & 9 & 4 & 9 & 3 & 2 \\
\hline Fabaceae & Acacia globulifera & 0 & 0 & 0 & 0 & 0 & 1 & 0 & 0 \\
\hline Fabaceae & Ateleia gummifera & 0 & 0 & 1 & 1 & 0 & 0 & 0 & 0 \\
\hline Fabaceae & Bauhinia divaricata & 48 & 9 & 6 & 1 & 0 & 0 & 1 & 0 \\
\hline Fabaceae & Caesalpinia gaumeri & 0 & 0 & 0 & 0 & 2 & 0 & 0 & 0 \\
\hline Fabaceae & Caesalpinia mollis & 1 & 1 & 11 & 1 & 8 & 1 & 7 & 0 \\
\hline Fabaceae & Caesalpinia vesicaria & 7 & 4 & 20 & 11 & 3 & 3 & 0 & 0 \\
\hline Fabaceae & Caesalpinia yucatanensis & 0 & 0 & 2 & 3 & 0 & 0 & 0 & 0 \\
\hline Fabaceae & Calliandra belizensis & 0 & 0 & 0 & 1 & 0 & 0 & 24 & 4 \\
\hline Fabaceae & Chloroleucon mangense & 6 & 1 & 0 & 0 & 0 & 0 & 0 & 0 \\
\hline Fabaceae & Diphysa carthagenensis & 3 & 5 & 0 & 3 & 2 & 2 & 3 & 0 \\
\hline Fabaceae & Erythrina mexicana & 1 & 0 & 0 & 0 & 0 & 0 & 1 & 0 \\
\hline Fabaceae & Gliricidia maculata & 0 & 1 & 2 & 0 & 2 & 0 & 1 & 1 \\
\hline Fabaceae & Haematoxylum campechianum & 0 & 0 & 3 & 2 & 0 & 0 & 0 & 1 \\
\hline Fabaceae & Havardia albicans & 0 & 0 & 11 & 5 & 6 & 2 & 1 & 1 \\
\hline Fabaceae & Leucaena leucocephala & 6 & 0 & 0 & 0 & 0 & 0 & 0 & 0 \\
\hline Fabaceae & Lonchocarpus castilloi & 9 & 10 & 69 & 23 & 34 & 32 & 2 & 0 \\
\hline Fabaceae & Lonchocarpus guatemalensis & 30 & 11 & 0 & 0 & 0 & 0 & 1 & 0 \\
\hline Fabaceae & Lonchocarpus rugosus & 109 & 8 & 39 & 10 & 74 & 11 & 1 & 0 \\
\hline Fabaceae & Lonchocarpus xuul & 180 & 145 & 351 & 330 & 222 & 281 & 37 & 0 \\
\hline Fabaceae & Lonchocarpus yucatanensis & 9 & 12 & 36 & 48 & 91 & 48 & 27 & 0 \\
\hline Fabaceae & Lysiloma latisiliquum & 19 & 16 & 32 & 12 & 93 & 25 & 2 & 0 \\
\hline Fabaceae & Mimosa bahamensis & 8 & 3 & 8 & 5 & 34 & 11 & 0 & 0 \\
\hline Fabaceae & Piscidia piscipula & 180 & 80 & 23 & 43 & 72 & 81 & 0 & 0 \\
\hline Fabaceae & Platymiscium yucatanum & 10 & 2 & 13 & 1 & 8 & 2 & 0 & 0 \\
\hline Fabaceae & Senna racemosa & 1 & 1 & 2 & 7 & 0 & 0 & 0 & 0 \\
\hline Fabaceae & Senna undulata & 1 & 0 & 0 & 1 & 0 & 0 & 0 & 0 \\
\hline Fabaceae & Swartzia cubensis & 13 & 3 & 18 & 6 & 7 & 2 & 6 & 0 \\
\hline Lamiaceae & Vitex gaumeri & 13 & 10 & 8 & 8 & 54 & 11 & 5 & 1 \\
\hline Lauraceae & Licaria campechiana & 0 & 0 & 0 & 0 & 0 & 0 & 2 & 0 \\
\hline Lauraceae & Licaria coriacea & 0 & 3 & 2 & 0 & 8 & 2 & 0 & 0 \\
\hline Lauraceae & Licaria peckii & 0 & 0 & 0 & 1 & 0 & 0 & 0 & 0 \\
\hline Lauraceae & Nectandra salicifolia & 77 & 49 & 167 & 79 & 181 & 71 & 103 & 13 \\
\hline Malpighiaceae & Byrsonima bucidifolia & 0 & 0 & 0 & 0 & 0 & 0 & 3 & 0 \\
\hline
\end{tabular}


APÉNDICE 1 (Continuación) / APPENDIX 1 (Continued)

\begin{tabular}{|c|c|c|c|c|c|c|c|c|c|}
\hline \multirow[b]{2}{*}{ Familia } & \multirow[b]{2}{*}{ Especie } & \multicolumn{2}{|c|}{ BSJ } & \multicolumn{2}{|c|}{ BSI } & \multicolumn{2}{|c|}{ BSA } & \multicolumn{2}{|c|}{ BT } \\
\hline & & $\begin{array}{l}\text { Sin } \\
\text { rebrote }\end{array}$ & $\begin{array}{l}\text { Con } \\
\text { rebrote }\end{array}$ & $\begin{array}{c}\text { Sin } \\
\text { rebrote }\end{array}$ & $\begin{array}{l}\text { Con } \\
\text { rebrote }\end{array}$ & $\begin{array}{c}\text { Sin } \\
\text { rebrote }\end{array}$ & $\begin{array}{l}\text { Con } \\
\text { rebrote }\end{array}$ & $\begin{array}{c}\text { Sin } \\
\text { rebrote }\end{array}$ & $\begin{array}{l}\text { Con } \\
\text { rebrote }\end{array}$ \\
\hline Malpighiaceae & Byrsonima crassifolia & 0 & 0 & 1 & 0 & 2 & 0 & 1 & 0 \\
\hline Malpighiaceae & Malpighia glabra & 5 & 5 & 35 & 7 & 46 & 23 & 21 & 1 \\
\hline Malpighiaceae & Malpighia lundellii & 0 & 0 & 2 & 4 & 2 & 8 & 5 & 0 \\
\hline Malvaceae & Guazuma ulmifolia & 0 & 0 & 0 & 1 & 0 & 0 & 0 & 0 \\
\hline Malvaceae & Hampea trilobata & 220 & 101 & 106 & 74 & 97 & 39 & 2 & 0 \\
\hline Malvaceae & Heliocarpus donnellsmithii & 7 & 1 & 0 & 0 & 0 & 0 & 0 & 0 \\
\hline Malvaceae & Pseudobombax ellipticum & 1 & 0 & 2 & 1 & 2 & 1 & 1 & 0 \\
\hline Meliaceae & Cedrela odorata & 21 & 5 & 0 & 0 & 0 & 0 & 0 & 0 \\
\hline Meliaceae & Swietenia macrophylla & 0 & 0 & 0 & 0 & 1 & 0 & 5 & 0 \\
\hline Meliaceae & Trichilia minutiflora & 0 & 0 & 0 & 0 & 0 & 0 & 2 & 0 \\
\hline Meliaceae & Trichilia pallida & 0 & 1 & 1 & 4 & 4 & 5 & 5 & 0 \\
\hline Menispermaceae & Hyperbaena winzerlingii & 0 & 0 & 1 & 0 & 0 & 0 & 0 & 2 \\
\hline Moraceae & Brosimum alicastrum & 1 & 0 & 0 & 0 & 1 & 1 & 39 & 1 \\
\hline Moraceae & Chlorophora tinctoria & 1 & 0 & 0 & 0 & 0 & 0 & 0 & 0 \\
\hline Moraceae & Ficus maxima & 0 & 0 & 0 & 0 & 1 & 0 & 0 & 0 \\
\hline Moraceae & Ficus obtusifolia & 1 & 0 & 1 & 1 & 1 & 1 & 1 & 0 \\
\hline Moraceae & Pseudolmedia spuria & 0 & 0 & 0 & 0 & 0 & 0 & 3 & 0 \\
\hline Moraceae & Trophis racemosa & 10 & 14 & 13 & 2 & 11 & 33 & 3 & 0 \\
\hline Myrtaceae & Calyptranthes schiedeana & 1 & 0 & 1 & 0 & 0 & 0 & 1 & 0 \\
\hline Myrtaceae & Calyptranthes paniculata & 0 & 0 & 0 & 1 & 0 & 0 & 0 & 0 \\
\hline Myrtaceae & Eugenia capuli & 4 & 0 & 14 & 9 & 38 & 6 & 3 & 0 \\
\hline Myrtaceae & Eugenia ibarrae & 52 & 38 & 205 & 90 & 345 & 63 & 55 & 7 \\
\hline Myrtaceae & Eugenia winzerlingii & 9 & 10 & 37 & 21 & 50 & 15 & 48 & 7 \\
\hline Myrtaceae & Myrcianthes fragrans & 0 & 0 & 0 & 0 & 1 & 0 & 0 & 0 \\
\hline Myrtaceae & Myrciaria floribunda & 0 & 0 & 21 & 5 & 49 & 6 & 32 & 2 \\
\hline Myrtaceae & Pimenta dioica & 0 & 0 & 1 & 0 & 0 & 0 & 11 & 0 \\
\hline Nyctaginaceae & Neea choriophylla & 4 & 4 & 0 & 1 & 16 & 11 & 21 & 0 \\
\hline Ochnaceae & Ouratea nitida & 2 & 0 & 0 & 0 & 13 & 1 & 3 & 0 \\
\hline Opiliaceae & Agonandra macrocarpa & 0 & 0 & 0 & 0 & 0 & 1 & 0 & 0 \\
\hline Opiliaceae & Agonandra ovatifolia & 1 & 0 & 2 & 2 & 0 & 1 & 4 & 0 \\
\hline Phyllanthaceae & Astrocasia tremula & 0 & 0 & 0 & 0 & 3 & 0 & 2 & 0 \\
\hline Phyllanthaceae & Phyllanthus graveolens & 2 & 2 & 0 & 0 & 0 & 0 & 0 & 0 \\
\hline Picramniaceae & Alvaradoa amorphoides & 10 & 1 & 9 & 0 & 0 & 0 & 0 & 0 \\
\hline Polygonaceae & Coccoloba acapulcensis & 0 & 0 & 2 & 1 & 3 & 3 & 4 & 4 \\
\hline Polygonaceae & Coccoloba barbadensis & 0 & 1 & 0 & 1 & 0 & 1 & 0 & 1 \\
\hline Polygonaceae & Coccoloba cozumelensis & 2 & 5 & 44 & 24 & 47 & 14 & 2 & 0 \\
\hline Polygonaceae & Coccoloba reflexiflora & 39 & 30 & 141 & 62 & 89 & 45 & 7 & 6 \\
\hline Polygonaceae & Gymnopodium floribundum & 1 & 8 & 6 & 15 & 24 & 50 & 6 & 21 \\
\hline Polygonaceae & Neomillspaughia emarginata & 14 & 24 & 34 & 43 & 84 & 78 & 0 & 0 \\
\hline Primulaceae & Bonellia flammea & 0 & 0 & 0 & 0 & 3 & 0 & 8 & 1 \\
\hline Primulaceae & Parathesis cubana & 6 & 1 & 6 & 0 & 7 & 5 & 3 & 0 \\
\hline Putranjivaceae & Drypetes lateriflora & 0 & 2 & 0 & 0 & 13 & 2 & 80 & 3 \\
\hline Rhamnaceae & Colubrina arborescens & 1 & 0 & 0 & 0 & 0 & 0 & 0 & 0 \\
\hline Rhamnaceae & Krugiodendron ferreum & 1 & 0 & 0 & 0 & 16 & 6 & 34 & 0 \\
\hline Rubiaceae & Cosmocalyx spectabilis & 0 & 2 & 6 & 5 & 0 & 1 & 5 & 0 \\
\hline Rubiaceae & Exostema caribaeum & 0 & 1 & 0 & 1 & 0 & 0 & 0 & 0 \\
\hline
\end{tabular}


APÉNDICE 1 (Continuación) / APPENDIX 1 (Continued)

\begin{tabular}{|c|c|c|c|c|c|c|c|c|c|}
\hline \multirow[b]{2}{*}{ Familia } & \multirow[b]{2}{*}{ Especie } & \multicolumn{2}{|c|}{ BSJ } & \multicolumn{2}{|c|}{ BSI } & \multicolumn{2}{|c|}{ BSA } & \multicolumn{2}{|c|}{ BT } \\
\hline & & $\begin{array}{l}\operatorname{Sin} \\
\text { rebrote }\end{array}$ & $\begin{array}{c}\text { Con } \\
\text { rebrote }\end{array}$ & $\begin{array}{c}\text { Sin } \\
\text { rebrote }\end{array}$ & $\begin{array}{c}\text { Con } \\
\text { rebrote }\end{array}$ & $\begin{array}{l}\mathrm{Sin} \\
\text { rebrote }\end{array}$ & $\begin{array}{c}\text { Con } \\
\text { rebrote }\end{array}$ & $\begin{array}{c}\text { Sin } \\
\text { rebrote }\end{array}$ & $\begin{array}{l}\text { Con } \\
\text { rebrote }\end{array}$ \\
\hline Rubiaceae & Exostema mexicanum & 2 & 14 & 0 & 0 & 0 & 0 & 0 & 0 \\
\hline Rubiaceae & Guettarda combsii & 55 & 39 & 195 & 43 & 107 & 23 & 1 & 0 \\
\hline Rubiaceae & Guettarda elliptica & 0 & 1 & 0 & 0 & 0 & 0 & 0 & 0 \\
\hline Rubiaceae & Guettarda gaumeri & 0 & 3 & 3 & 8 & 3 & 0 & 2 & 4 \\
\hline Rubiaceae & Hamelia patens & 4 & 2 & 3 & 0 & 0 & 0 & 0 & 0 \\
\hline Rubiaceae & Machaonia lindeniana & 8 & 7 & 11 & 15 & 6 & 2 & 1 & 0 \\
\hline Rubiaceae & Randia aculeata & 8 & 8 & 37 & 13 & 33 & 18 & 5 & 1 \\
\hline Rubiaceae & Randia longiloba & 2 & 3 & 1 & 3 & 3 & 4 & 4 & 6 \\
\hline Rubiaceae & Simira salvadorensis & 1 & 2 & 4 & 0 & 3 & 1 & 3 & 1 \\
\hline Rutaceae & Amyris elemifera & 0 & 0 & 0 & 0 & 0 & 0 & 8 & 1 \\
\hline Rutaceae & Esenbeckia berlandieri & 45 & 16 & 0 & 0 & 0 & 0 & 1 & 0 \\
\hline Rutaceae & Zanthoxylum juniperinum & 0 & 0 & 0 & 1 & 2 & 1 & 1 & 0 \\
\hline Salicaceae & Casearia emarginata & 1 & 0 & 0 & 0 & 10 & 12 & 2 & 0 \\
\hline Salicaceae & Laetia thamnia & 0 & 2 & 2 & 1 & 11 & 0 & 21 & 1 \\
\hline Salicaceae & Samyda yucatanensis & 0 & 0 & 2 & 0 & 5 & 0 & 6 & 1 \\
\hline Salicaceae & Xylosma flexuosa & 0 & 0 & 1 & 0 & 1 & 0 & 1 & 0 \\
\hline Salicaceae & Zuelania guidonia & 18 & 21 & 37 & 20 & 18 & 17 & 0 & 0 \\
\hline Sapindaceae & Allophylus cominia & 32 & 32 & 31 & 21 & 48 & 25 & 0 & 0 \\
\hline Sapindaceae & Cupania belizensis & 4 & 1 & 24 & 3 & 12 & 4 & 0 & 0 \\
\hline Sapindaceae & Exothea diphylla & 0 & 0 & 1 & 0 & 3 & 3 & 9 & 0 \\
\hline Sapindaceae & Matayba oppositifolia & 0 & 0 & 4 & 2 & 0 & 1 & 0 & 0 \\
\hline Sapindaceae & Melicoccus oliviformis & 2 & 2 & 0 & 0 & 8 & 2 & 34 & 1 \\
\hline Sapindaceae & Thouinia paucidentata & 18 & 23 & 47 & 35 & 78 & 36 & 23 & 9 \\
\hline Sapotaceae & Chrysophyllum argenteum & 0 & 0 & 1 & 0 & 1 & 0 & 33 & 0 \\
\hline Sapotaceae & Chrysophyllum mexicanum & 21 & 18 & 11 & 8 & 22 & 5 & 2 & 0 \\
\hline Sapotaceae & Manilkara zapota & 0 & 0 & 2 & 0 & 19 & 4 & 62 & 4 \\
\hline Sapotaceae & Pouteria campechiana & 0 & 1 & 1 & 2 & 2 & 0 & 2 & 0 \\
\hline Sapotaceae & Pouteria reticulata & 0 & 0 & 4 & 0 & 99 & 4 & 222 & 5 \\
\hline Sapotaceae & Sideroxylon obtusifolium & 0 & 1 & 2 & 1 & 2 & 2 & 0 & 0 \\
\hline Sapotaceae & Sideroxylon rotundifolium & 2 & 0 & 3 & 1 & 1 & 0 & 0 & 0 \\
\hline Sapotaceae & Sideroxylon salicifolium & 8 & 9 & 4 & 4 & 6 & 2 & 2 & 0 \\
\hline Simaroubaceae & Simarouba amara & 0 & 0 & 0 & 0 & 4 & 0 & 1 & 0 \\
\hline Solanaceae & Cestrum nocturnum & 1 & 1 & 0 & 0 & 0 & 0 & 0 & 0 \\
\hline Urticaceae & Cecropia peltata & 53 & 17 & 12 & 4 & 0 & 0 & 0 & 0 \\
\hline Verbenaceae & Rehdera trinervis & 4 & 3 & 0 & 0 & 9 & 1 & 2 & 0 \\
\hline Zygophyllaceae & Guaiacum sanctum & 0 & 0 & 0 & 0 & 0 & 0 & 9 & 6 \\
\hline
\end{tabular}

*=Especie donde no se obtuvo datos de campo, ni con el informante clave y revisión de literatura sin datos. 